\title{
Claude Simon et Sartre : les premiers romans
}

Jean-François Louette

\section{OpenEdition}

Journals

Édition électronique

URL : http://journals.openedition.org/ccs/570

DOI : $10.4000 /$ ccs. 570

ISSN : 2558-782X

\section{Éditeur :}

Presses universitaires de Rennes, Association des lecteurs de Claude Simon

\section{Édition imprimée}

Date de publication : 30 avril 2007

Pagination : 63-85

ISBN : 9782354120122

ISSN : 1774-9425

\section{Référence électronique}

Jean-François Louette, "Claude Simon et Sartre : les premiers romans », Cahiers Claude Simon [En ligne], 3 | 2007, mis en ligne le 20 septembre 2017, consulté le 19 avril 2019. URL : http:// journals.openedition.org/ccs/570; DOI : 10.4000/ccs.570 


\title{
Claude Simon et Sartre : les premiers romans
}

\author{
Jean-François FOUETTE*
}

Claude Simon et Sartre : j'ai commencé mon enquête sur ce dialogue entre deux très fortes personnalités littéraires par une désillusion. Le livre de Betty T. Rahv, From Sartre to the New novel $\backslash$ ignore radicalement le nom de Claude Simon, et privilégie Robbe-Grillet. En revanche j'ai beaucoup appris en découvrant un article, très synthétique et très précis, d'Alastair Duncan, "Simon and Sartre », paru en $1985^{2}$. Vingt ans après, il n'y a guère à y ajouter. L'hypothèse initiale de ce travail est qu'une " manière nécessairement incomplète de comprendre la première phase de Simon est de le voir qui émerge graduellement de l'ombre de Sartre », personnage en effet fort encombrant pour qui entre dans le champ littéraire dans les années quarante. La conclusion d'Alastair Duncan est que Simon «n'a pas pardonné à Sartre de l'avoir égaré, non seulement dans Gulliver [roman social, qui met en scène les comptes qu'il y a à régler dans l'immédiat après-guerre], mais aussi durant le long apprentissage pendant lequel, par essais et erreurs, il a découvert comment réviser sa conception de ce qu'est un roman, ou de ce qu'il devrait chercher

Université Paris IV-Sorbonne.

Port Washington, Kennikat Press, 1974.

${ }^{2}$ Review of contemporary fiction, vol. 5, $\mathrm{n}^{\circ} 2$, Spring 1985, p. 90-95; il s'agit d'un numéro spécial sur Claude Simon. 
à être ». Bref : c'est pour avoir d'abord trop suivi Sartre que Simon - qui possédait, me dit-on, toute la collection des Temps modernes! - lui a ensuite jeté beaucoup de pierres. J'ai d'abord pensé me borner à traduire cet article... Tout en reconnaissant la justesse de cette hypothèse, je tenterai de lui apporter quelques compléments. - J'ai encore eu la bonne fortune de dénicher dans ma bibliothèque le livre de Nelly Wolf, Une littérature sans histoire. Essai sur le Nouveau Roman (Droz, 1995), et de me voir prêter par Brigitte Combe l'ouvrage de Dominique Viart, Une mémoire inquiète. "La Route des Flandres » de Claude Simon (PUF, 1997), où un riche chapitre est consacré à « La question de l'engagement ». Enfin, Nathalie Piégay-Gros a insisté de son côté sur la dénonciation chez Simon de l'écriture sans risques, dégagée, de l'après-coup, à la Churchill ${ }^{1}$ : la relation avec Sartre n'est pas seulement d'opposition. C'est pourtant par là que l'on commencera.

\section{Une opposition frontale}

Sartre a préfacé en 1957 le Portrait d'un inconnu de Sarraute. Mais ensuite s'est produit chez lui un éloignement sélectif à l'égard du Nouveau Roman. Interviewé par Madeleine Chapsal, en 1960, à la question "Quels sont les écrivains contemporains qui vous intéressent ? », il répond ainsi : " Il y a des écrivains de grand talent : Butor, Beckett. Je m'intéresse beaucoup aux ouvres de Robbe-Grillet, de Nathalie Sarraute. Mais, si nous envisageons leurs œuvres du point de vue de la totalité, je vous dirai qu'il y en a un seul, en France, pour se formuler clairement le problème et répondre aux exigences du tout : c'est Butor $»^{2}$. Et de faire l'éloge de Degrés. Où est Claude Simon? Sartre l'a sélectionné par son omission.

Chez Simone de Beauvoir, les lois du champ littéraire, qui ne prévoient guère d'affection entre deux générations d'écrivains qui se succèdent, se manifestent avec plus de virulence. Dans La Force des

1 « Mélancolie du montage. Le Jardin des Plantes de Claude Simon », L'Engagement littéraire, Emmanuel Bouju éd., Presses Universitaires de Rennes, 2005, p. 283292.

2 Repris dans Les Ecrivains en personne, et aussi en ouverture de Situations, IX, Gallimard, 1972, ici p. 17. 
choses, qui paraît en avril 1964, elle se plaint du fait que le Nouveau Roman, de son époque, n'a « à peu près rien exprimé ». Sarraute ? " Reprenant à son compte le vieux psychologisme français, elle décrit avec talent l'attitude paranoïaque de la petite bourgeoisie, comme si elle constituait l'immuable nature de l'homme ». RobbeGrillet? Pour lui « l'apparence est tout, défense de la dépasser». Butor? Chez lui « la littérature se prend elle-même pour objet » : il insiste "sur l'inadéquation spatiale et temporelle du récit et de la réalité » (on voit que le propos est moins sévère). Vient cependant le coup de grâce, auquel Beckett échappe : «Dans l'ensemble, une des constantes de cette littérature, c'est l'ennui »1 ${ }^{1}$. Et Claude Simon ? Simone ne daigne pas nommer Simon. Pourtant il me semble qu'il est visé par cette phrase perfide : «Tantôt, choisissant de ne rien dire, on masque par des contorsions formelles l'absence de contenu, pastichant Faulkner et Joyce qui avaient inventé des moyens inédits de dire quelque chose de neuf ».

Beauvoir oublie d'en appeler à Conrad... et à Proust. C'est cette ombre auguste que Sartre à son tour convoquera pour offusquer Simon, dans une interview donnée à Clarté, mensuel de l'Union des étudiants communistes, en mars-avril 1964 : «Considérons le nouveau roman, que j'aime bien d'ailleurs, qu'apporte-t-il de nouveau par rapport à d'autres ? ». À cette question redoutable, Sartre répond par l'envers de son omission de 1960 ; il ne retient que le cas de Claude Simon : «Prenons Claude Simon, par exemple. Il écrit sur le temps, la mémoire. Au fond, que montre-t-il de plus que Proust? Vous me direz, il y a l'arrangement différent. Mais nous parlons de signes, et l'arrangement ne change quelque chose que si le contenu a changé. Or, je ne vois pas qu'il y ait de contenu différent entre lui - Claude Simon - et Proust. Je dis donc que je me trouve en présence de tentatives habiles, intéressantes, mais qui ne changent pas le contenu depuis Proust ». Cet argument paraît pour le moins sommaire. On pourrait lui donner un peu plus de profondeur en passant par l'article qu'en juillet 1939, dans la $N R F$, Sartre consacrait à « La temporalité chez Faulkner ». Il rapprochait le temps de Faulkner et 
celui de Proust, deux mondes romanesques dans lesquels les personnages jamais ne prévoient, deux mondes qui ignorent la dimension de l'avenir : or l'héritage de Faulkner, Simon ne l'a, je crois, jamais répudié. On voit alors la chaîne transitive qui adoucit un peu, parce qu'elle l'explique, la polémique de 1964 : si Sartre a (un peu) raison de dire que Simon fait du Proust, c'est parce qu'il (Simon) fait du Faulkner, qui lui-même faisait (un peu) du Proust...

Tout cela est (un peu) approximatif. Renversons plutôt le point de vue. De Sartre, que pensait Claude Simon ? Certes peu de bien. On pourrait énumérer nombre de textes à charge ${ }^{1}$. Je préfère m'attarder sur l'un d'entre eux : la réaction scandalisée de Simon, dans L'Express du 28 mai 1964, à l'interview de Sartre par Jacqueline Piatier, dans Le Monde du 18 avril de la même année, dans laquelle figure cette déclaration célèbre : "En face d'un enfant qui meurt, La Nausée ne fait pas le poids »- proposition qui pour Sartre voulait dire « que signifie la littérature dans un monde qui a faim ? ». Ce numéro de L'Express porte en couverture une photo de Charles de Gaulle, et pour titre de une, en capitales: ATTENTION DE GAULLE S'ENNUIE. A-t-il trouvé une distraction dans les réponses à Sartre que le journal demande à Yves Berger, 30 ans, et à Claude Simon, 50 ans ? De celle d'Yves Berger, par charité, nous ne dirons rien (retenons cependant ceci : il fait observer que c'est précisément sa gloire

1 Ainsi : 1. l'interview de 1960 par Madeleine Chapsal ; 2. écrite lors de l'affaire de la discussion publique de l'automne 1964, organisée par Clarté à la Mutualité, débat auquel Simon renonce parce que Sartre et Beauvoir refusent de dialoguer avec Kostas Axelos (voir Beauvoir, Tout comptefait, Gallimard, 1972, p. 138), la «Lettre ouverte à l'Union des étudiants communistes » (L'Express) ; 3. un passage des Corps conducteurs (1971), qui, sous le titre «Dialogue entre parlementaires et écrivains ", met en scène de façon satirique les thèses sartriennes, déplacées jusqu'en Amérique du Sud - passage très précisément analysé par Alastair Duncan à la fin de son article ; 4. l'attaque brutale dans le Discours de Stockholm, en 1985, qui confond dans le même mépris Ta Condition humaine, L'Espoir, et Tes Chemins de la liberté ; 5. la diatribe contre Sartre dans l'entretien accordé à Bernard-Henri Lévy, paru dans Tes Aventures de la liberté, Grasset, 1991 ; 6. la page aigrelette du jardin des plantes (Minuit, 1997, p. 338) sur Sartre faisant jouer Les Mouches à Paris en 1943, injuste car elle oublie tout le plaidoyer pour la liberté d'Oreste, et donc le sens même de la pièce, qui n'a pas échappé aux spectateurs de l'époque. 
littéraire qui donne leur poids aux mots de Sartre). Et quant à Claude Simon ? Signalons tout d'abord que l'année 1964 avait de quoi raviver les plus légères allergies à Sartre puisque c'est une véritable salve de ses livres qui explose en cet an de grâce : Les Mots en janvier, puis Situations, $I L$, et $V$, et $V I$, sans oublier une réédition de Qu'est-ce que la littérature? dans la collection Idées. Ajoutons que Simon a dû aussi être ulcéré par l'interview (toujours de Sartre...) parue dans Clarté (en mars-avril) ; aussi, au joli mois de mai, comprend-il les propos de Sartre comme une négation radicale de la valeur de la littérature. Il lui oppose quatre arguments principaux, dans un texte qui n'est pas une interview, mais une réponse écrite et mûrement pensée. Arguments que je reprends, dans un désordre concerté.

1. Le premier provient de l'expérience qu'a Simon de l'écriture le langage n'y sert pas d'instrument qui aurait pour mission d'exprimer une idée préexistante, mais il «déplie ses propres lignes de force ", si bien qu'écrire est " une sorte d'exploration, une hasardeuse aventure ". C'est donc la distinction entre prose (langageinstrument) et poésie (langage devenant sa propre fin) établie dans Qu'est-ce que la littérature? qui est visée. Dans le roman selon Simon, le langage fonctionne de manière poétique. Je ne crois qu'à demi à la sincérité de cet argument : il me semble que le plus souvent le formalisme chez Simon est tactique - c'est précisément une manière d'échapper à Sartre.

3. Le troisième argument consiste à demander : "depuis quand pèse-t-on cadavres et littérature sur une même balance ?» - c'est-àdire à poser en principe l'hétérogénéité radicale de deux ordres.

4. Le dernier argument est de fait: prisonnier de guerre, lui, Claude Simon, a souffert de la faim extrême, mais « n'importe quel livre [lui] était précieux ", comme à ses compagnons de misère, même à demi incultes. Argument en partie sophistique : Sartre parle de la mort advenue d'un enfant (qui ne peut donc plus lire, ou n'a jamais eu le temps d'apprendre), Simon du dépérissement qui conduit à l'agonie, mais sans encore empêcher de lire.

2. Le deuxième argument repose sur une analyse paradoxale du 
célèbre tableau de Picasso, Guernica. Il me semble que Simon répond ici à un texte de Sartre, où figurait le même exemple. Ce texte, intitulé «Le Peintre sans privilèges », et consacré à Robert Lapoujade, n'avait pas été publié moins de trois fois en 1961: comme préface au catalogue de l'exposition Lapoujade, Peintures sur le thème des émeutes, Triptyque sur la torture, Hiroshima, puis en fragments dans France-Observateur, et enfin, intégralement, dans Médiations, $\mathrm{n}^{\circ} 2$. Il fut ensuite repris dans Situations, $\boldsymbol{I} \boldsymbol{V}^{1}$. Or Sartre en passait par Picasso. L'écrivain part de la question suivante : l'indignation d'un peintre peut-elle descendre jusqu'à son pinceau ? Ce qui revient à demander si un peintre peut s'engager, et peindre efficacement ${ }^{2}$. Or la réponse de Sartre est... négative : la peinture meurt si elle est assignée à la figuration de l'horreur, inféodée à «la Bonne Pensée », asservie «à des fins étrangères ». Cependant, l'option inverse (faire disparaître l'horreur sous les splendeurs, comme Titien, selon Sartre) revient à ne pas rendre compte du mal et de la souffrance. On voit le dilemme : « trahir la peinture sans grand profit pour la Morale, ou, si l'œuvre, en dépit de tout, paraissait belle, trahir pour la Beauté la colère ou la peine des hommes. Trahison partout $»$. Seuls deux peintres auraient su échapper à ce dilemme : Goya, et Picasso. Et Sartre alors de consacrer une belle page à Guernica, pour dire en substance que dans cette toile Picasso a réussi à la fois à être engagé (" inoubliable révolte, commémoration d'un massacre») et à faire œuvre belle, parce que s'entr'expriment la désintégration des hommes sous les bombes, et la désintégration des figures dans la recherche esthétique du peintre, c'est-à-dire dans sa manière "explosante-fixe " (c'est Sartre qui cite le mot de Breton). - Laissons maintenant le Sartre de 1961 : il va tenter de montrer comment la peinture nonfigurative (celle de Lapoujade) peut être engagée, sans donner à voir la torture, mais en faisant éprouver, par un " concert de stries ", son sens inanalysable. Et revenons au Claude Simon de 1964, qui entend bien donner à Sartre une leçon de peinture à propos de Guernica. Selon lui, à partir certes d'une « réalité horrible", et «mû par

1 Voir M. Contat et M. Rybalka, Les Écrits de Sartre, Gallimard, 1970, p. 361.

2 Voir George H. Bauer, "Peinture et engagement ", Sartre et les arts, Obliques, $\mathrm{n}^{\circ} 24-25,1980$, p. 263-266. 
l'indignation » (on voit que revient le mot même qu'avait employé Sartre), Picasso peint « un tableau où tout n'est qu'ordre, équilibre et beauté » : pas de dissonance, sobriété des couleurs, "la froide pâleur du marbre ", une multiplication de "références au musée » (par ex. à un tableau de Poussin, Lejugement de Salomon, qu'on peut voir au Louvre), si bien que le Mal devient « délectation » (tel était le but que Poussin assignait à l'art du peintre). On peut n'être pas convaincu, et continuer à penser que la violence elle aussi s'exprime dans Guernica, comme d'ailleurs dans les romans de Simon, dont la dé-composition trouverait un bel emblème dans cette image éclatée de la guerre que donne Guernica, avec, pour centre, à peu près à la place de Salomon (mais en même temps significativement décentré)... un cheval souffrant et hennissant! Du |ugement de Salomon au cheval de Simon (et Picasso), on passe de la Justice au chaos, sans pour autant quitter le champ de l'art.

Donc : conflit ouvert, rien à ajouter ? Non pas. L'attitude de Simon est pour une part stratégique : elle est «le moyen de se désengager de la mouvance sartrienne et existentialiste " avec laquelle le Nouveau Roman " partageait à l'origine bon nombre de ses présupposés esthétiques ». C'est cette proximité initiale qui expliquerait la fureur de Simon contre Sartre. On le verra en relisant ses trois premiers romans, et $L a$ Corde raide, qui n'est pas donnée pour un roman, ni dans l'édition originale ni dans celle qui parut chez Minuit ${ }^{2}$.

\section{Des liens précoces et profonds}

Robbe-Grillet a fait le point en 1979, lors du premier colloque de Cerisy consacré à Sartre, sur son rapport à Sartre ${ }^{3}$. Cette intervention qui s'intitule "Sartre et le Nouveau Roman » (en fait il s'agit plutôt d'un : Sartre et moi, Robbe-Grillet) est le lieu d'un aveu de poids : Robbe-Grillet y présente La Nausée comme un " déclencheur d'écriture " pour lui, et son dessein (au moins en partie) comme

Nelly Wolf, ouvr. cité, p. 193.

2 Je remercie Brigitte Combe de m'avoir communiqué ce texte, ainsi que Le Tricheur.

3 Voir Etudes sartriennes, II-III, Publidix, 1986. 
celui d'« essayer d'écrire ce roman qui devait sauver Roquentin». Il reprendra la question dans Le Miroir qui revient. - De son côté Michel Butor raconte, dans un entretien, qu'une conférence de Sartre en 1944, intitulée «Une technique sociale du roman», lui permit d'entendre parler, pour la première fois, de Virginia Woolf, de Dos Passos et de Faulkner, et qu'une « bonne partie de la problématique de [s]es propres romans s'est développée à partir des réflexions qui [lui] sont venues lors de cette conférence », qui portait sur la manière de « sortir du petit roman centré sur un ou deux personnages 1 . - Claude Simon en revanche n'a jamais reconnu l'importance de Sartre pour la genèse de son œuvre romanesque. Elle existe pourtant. Et plus forte peut-être que pour Butor et Robbe-Grillet.

On se souvient du mot célèbre de Sartre : « une technique romanesque renvoie toujours à la métaphysique du romancier. La tâche du critique est de dégager celle-ci avant d'apprécier celle-là $»^{2}$. Pour diminuer l'ire des mânes de Claude Simon, commençons quand même par la technique.

\section{Technique}

Sur le plan de la technique romanesque, je reprends l'hypothèse (déjà formée par Nelly Wolf) que Claude Simon a été très sensible aux analyses de Sartre dans les années trente. Il avait pu les lire à la fois dans La Nausée, et dans cette salve d'articles brillants, parus dans la $N R F$, qui ont fait beaucoup de bruit en 1938-1939 : ils portaient sur Faulkner («Sartoris par William Faulkner», février 1938), Dos Passos («À propos de John Dos Passos et de 1919», août 1938), Mauriac (« M. François Mauriac et la liberté », février 1939), Faulkner encore ( À propos de Le Bruit et la Fureur : la temporalité chez Faulkner», juillet 1939). Faut-il leur adjoindre la fameuse « Explication de L'Étranger» (Cahiers du Sud, février 1943) ? Autant d'articles vite célèbres, qui seront réunis dans Situations, $I$ en octobre 1947.

1 Obliques, $\mathrm{n}^{\circ}$ 24-25, Sartre et les arts, 1981, p. 67.

2 «À propos de Le Bruit et la Lureur. La temporalité chez Faulkner », Situations, I, Gallimard, 1947, rééd. 1978, p. 66. 
Repartons de La Nausée, qui paraît en avril 1938. Roquentin, qui a lu comme Sartre tel fragment de Valéry, "La vie est un conte » (publié dans la $N R F$ en 1933, en volume dans Suite en 1934), note à quel point l'existence est sans ordre, alors que le récit - en particulier historique, mais aussi romanesque - lui en impose un. Le narrateur de La Nausée, en même temps qu'il renonce à sa biographie du marquis de Rollebon, produit une critique de la causalité narrative, qui transforme le post hoc en propter hoc. Désordre de l'existence, ordre causal et finalisant à tort imposé par le récit, et en particulier par les historiens : c'est cette contradiction que Simon ne va cesser d'explorer.

Il l'avait retrouvée dans les articles de 1938-1939, où Sartre fait un pas de plus, et oppose fortement le roman et le récit. Ce dernier est explicatif, il s'en remet à "l'ordre des causes » que voile à peine l'ordre chronologique. Claude Simon reprendra la même idée plus d'une fois, en particulier dans Les Géorgiques, en 1981, à propos de George Orwell songeant à tirer de son expérience en Espagne un récit cohérent :

\begin{abstract}
Peut-être espère-t-il qu'en écrivant son aventure il s'en dégagera un sens cohérent. Tout d'abord le fait qu'il va énumérer dans leur ordre chronologique des événements qui se bousculent pêle-mêle dans sa mémoire ou se présentent selon des priorités d'ordre affectif devrait, dans une certaine mesure, les expliquer. Il pense aussi peut-être qu'à l'intérieur de cet ordre premier les obligations de la construction syntaxique feront ressortir des rapports de cause à effet. $(G$, 310-311)
\end{abstract}

Cohérence explicative et causale : voilà les traits mêmes du récit selon Sartre (qui lui-même s'inspire de Ramon Fernandez).

Il faut bien en distinguer le roman, qui suppose la liberté des personnages, laquelle n'est pas séparable du privilège du présent. En effet, selon Sartre, « le roman se déroule au présent, comme la vie », si bien que « dans le roman les jeux ne sont pas faits » - tel est l'axiome posé au début de l'article sur Dos Passos. Les romans de Faulkner, note encore Sartre, accordent un privilège tout particulier au présent. Un présent qui surgit, soudain, «catastrophique »; un 
présent caractérisé par "l'enfoncement», c'est-à-dire fait d'une «sorte de mouvement immobile», de «jaillissements figés» 1 . N'est-ce pas cet effet même que Simon cherchera en recourant à ses fameux participes présents?

Sartre insiste sur "la technique du désordre" que pratique Faulkner, "ces inextricables mélanges de présent et de passé »; le romancier américain a "cassé le temps de son histoire " et en a «brouillé les morceaux $»^{2}$; il propose une temporalité non chronologique, un «temps sans horloge». D'où, note Sartre, le geste de Quentin qui brise sa montre : on retrouvera une montre cassée dès le tout début du premier roman de Simon, Le Tricheur (Louis jette la montre de son père tué à la guerre, et qui ne marche plus). Si l'horloge est cassée, le roman devrait pouvoir devenir ce que programme une belle formule de Sartre à propos de Dos Passos "le dévidage d'une mémoire brute et criblée de trous", dévidage qui se fait dans un style dont l'addition est la règle, pour déjouer la cohérence explicative. On songe à ce qu'écrit Simon, en 1957, au tout début du Vent. Tentative de restitution d'un retable baroque: " une addition de brèves images $» . .$. « tout cela vague, plein de trous, de vides»... «les débris, dispersés, incomplets, d'un miroir»... non pas une cohérence mais notre « errance »... $(V ., 3-4)$.

Dans La Nausée s'exprimait déjà la volonté de dégager le roman du modèle aristotélicien de l'intrigue se refermant sur elle-même, alors que la vie est sans commencement ni fin bien marqués. Simon notera dans La Corde raide, en 1947, un reproche qu'on lui a adressé, et qui révèle sans doute l'influence de ces célèbres pages de La Nausée «Ce genre d'histoires sans commencement ni fin, le public n'aime pas ça.» $(C R, 89)$. Autre rupture avec un modèle aristotélicien: selon Sartre, Faulkner écrit avec Le Bruit et la Fureur un roman sans " nœud » de l'action : "rien n'advient, l'histoire ne se déroule pas ». Pourquoi ? Parce qu'il est bien difficile d'en venir à un Acte. Sartre propose, dans son article sur Sartoris, une définition du roman par l'Acte insaisissable : «les Actes font l'essentiel du roman: on les

2 Ibid., p. 11 et 65 . 
prépare avec soin et lorsqu'ils arrivent, [...] ils nous glissent entre les doigts. On n'a plus rien à en dire ». C'est un problème - celui du caractère fuyant, et, à la limite, innommable de l'acte - que Simon retrouvera dans Le Tricheur (paru en 1945).

Critique de la cohérence explicative et causale imposée par le récit, privilèges accordés au présent, à un temps sans horloge, à une brisure de la temporalité où se mêlent en désordre présent et passé, problème de l'acte fuyant: il me semble incontestable que les textes de Sartre, en 1938-1939, définissent un art du roman et un paradigme romanesque, bref un type de roman à faire, qui ont exercé sur Simon (comme sur Butor et Robbe-Grillet) une très grande influence. Ce n'est donc point par hasard que la première réception de ce qui n'était pas encore baptisé Nouveau Roman y vit un roman phénoménologique ${ }^{1}$.

\section{Métaphysique}

Avec la question du temps on touchait déjà à la métaphysique. Mais repartons du Tricheur.; donné pour achevé en avril 1941, à la dernière page du roman (cette date est-elle exacte ? Il serait important d'en être sûr...).

Dans le deuxième chapitre apparaît un peintre, Gauthier, dont les premières pensées sont pour indiquer que ni les choses ni l'homme n'ont de raison d'être. Un peu plus loin il reviendra sur ce thème : il n'y a «ni raisons ni explications » (Tir., 108). Dès lors, comme le pense le protagoniste du premier chapitre, Louis, «Tout ça est parfaitement idiot. Un jeu : je gagne, il gagne... Gagner quoi ? Conception de l'existence sous l'optique du pari mutuel» (46). Du coup, sans autre raison que de se manifester à lui-même le pouvoir de sa volonté, Gauthier fait exprès de se flanquer par terre en vélo... Par cet acte cycliste et gratuit il semble se situer du côté de Lafcadio dans Les Caves du Vatican. Plus tard, dans un fragment de psycho-récit consacré à Louis, il sera question des « [p]oissons lumineux portant en eux-mêmes leur lumière, errant au sein des ténèbres ", etc. (218). On pense aux propos de Vincent, dans Les Faux monnayeurs, sur les 
« appareils photogéniques » des poissons de profondeurs (Première partie, ch. XVII).

Se met en place un dispositif qu'on pourrait nommer le dispositif Gide/Sartre. Car Louis veut échapper au hasard et à la gratuité, au statut de bout de bois qui flotte dans l'eau, en affermissant sa volonté et en posant un acte : «Je pense que tôt ou tard, il faudra faire la preuve que l'on a été, que l'on est, autre chose qu'une suite de loteries... » $(T r .:, 41)$. Voilà dessinée la problématique qui explique le titre: peut-on corriger le hasard, le conduire... Ou bien en demeure-t-on la proie passive ? Louis vit l'« [a]nxieuse interrogation des hommes à la recherche de leurs possibles » (218); et il désire échapper à cette errance par un acte (219). À la fin du roman, on le trouve sur un pont, figé face au fleuve, et décidant de son acte : « Mais je l'ai décidé. Un acte» (238). Un acte contre un salaud, acte qui serait une espèce de génération spontanée. Il s'agira de tuer un prêtre d'un coup de brique. Notamment à cause, semble-t-il, de la chaleur: et Maurice Nadeau ne manquera pas de rapprocher Le Tricheur de L'Étranger (qui ne paraît cependant qu'en 1942).

On aura noté l'intervention du terme existence (" l'existence sous l'optique du pari mutuel»). Celui de salaud (qui figure, comme nul ne l'ignore, déjà dans La Nausée) apparaît quelques pages plus loin, trois fois en un seul paragraphe ( $T r$., 50), à propos d'un prêtre aux mains baladeuses, insulte qu'adressée à un tel personnage Sartre n'eût certes pas désavouée. Plus loin encore on verra par les rues errer des papiers sales (109), comme dans le roman de 1938. Le lecteur du Tricheur rencontrera aussi le mot d'absurde (" Ou si tout cela ne se réduit pas seulement à une question de paroles plaquées sur l'absurde. » [213] ; « [...] la vie est une chose absurde terminée par une autre absurdité qui est la mort. » [243]), mot qui lui aussi figurait dans La Nausée ("l'absolu ou l'absurde », avec un ou d'identité). «J'ai d'ailleurs beaucoup trop lu», pense Louis (218). Sans doute ce personnage a-t-il, comme son auteur, lu La Nausée.

Il y aura même, dans Le Tricheur, un garçon de café

C'était une véritable caricature de garçon de province, comme on en voit sur les dessins d'avant-guerre: deux mèches 
soigneusement cirées, ramenées sur son crâne chauve, et une superbe paire de moustaches frisées.

— Amédée ou le parfait garçon de café, dit Lion. (Tr., 110)

Et encore de belles phrases sur la honte ( La honte part des cheveux et coule sur le visage et les membres. » [216]), et même sur le visqueux (« la tiède et visqueuse enveloppe de la honte » [220], « cette visqueuse tunique collée à la peau » [237], avec le souvenir d'Hercule, figure mythique chère à Claude Simon)! Ajoutons que le thème de l'acte apparaît dans un dialogue de Louis avec un personnage nommé Philippe, qui se fait exclure du peloton pour les élèves officiers... Ironie des choses, Philippe est aussi le prénom d'un jeune homme qui dans Les Chemins de la liberté choisit de déserter. Le héros de la trilogie sartrienne, Mathieu Delarue, éprouve fortement que sa liberté s'oppose au hasard: "Toute sa liberté venait de refluer sur lui. Il pensa : " Non, non, ce n'est pas pile ou face. Quoi qu'il arrive, c'est par moi que tout doit arriver " $»^{1}$. Plus tard, Mathieu lui aussi se trouve sur un pont - le Pont-Neuf - face à sa liberté, libre pour rien, tenté par le suicide, cet « acte unique, incomparable»; mais, « tout à coup, il décida de ne pas le faire $»^{2}$, avant de décider que le sort d'un jeune déserteur - Philippe, précisément - le concerne.

Vous sentez bien que je dérape. Je suis en train de lire Le Tricheur comme si Claude Simon avait pu connaître (pour le garçon de café, la honte, le visqueux) L'Être et le Néant, qui ne paraît qu'en 1943, ainsi que L'Age de raison, Le Sursis qui ne sont publiés qu'en septembre 1945 (même si deux fragments de L'Âge de raison sont donnés en 1943 dans Messages et dans L'Arbalète). Il faut évidemment faire bien attention à la chronologie : dans Le Tricheur, c'est dès 1941 que Claude Simon réfléchit sur l'existence en opposant le hasard et la volonté. Ce que Sartre va faire aussi en 1943, dans L'Être et le Néant, et en 1945, dans L'Âge de raison et Le Sursis. Le problème est que Simon ne pourra pas, dans l'immédiat après-guerre, faire valoir l'antériorité de sa vision, ou de sa réflexion. Il débute, mais pas Sartre. Lui publie aux Éditions du Sagittaire, Sartre chez Gallimard. Sartre joue sur 
deux registres : philosophe, il donnera du débat hasard/action une version simplifiée, grand public et à grand succès dans $L$ 'existentialisme est un humanisme, en même temps que romancier, il publie les deux premiers volumes des Chemins de la liberté. Bref : ce qui expliquerait la grande fureur que Simon n'a jamais cessé d'exprimer à l'endroit de Sartre, ce serait qu'il a vécu en 1945, du fait de Sartre, un vol de thèmes, et de succès. En un sens, il se passe entre les deux ce qui s'est passé pour Queneau avec Céline : Queneau achève Le Chiendent en novembre 1932, roman dont l'un des enjeux est de faire de la philosophie en langage parlé ; il voit paraître Le Voyage au bout de la nuit en octobre de la même année... et écrit alors à Bataille qu'il est fichu, que Céline lui a volé toute sa gloire à venir. Y a-t-il une lettre analogue de Simon à un ami, sur ce voleur que serait Sartre?

Le deuxième chapitre du Tricheur se termine sur un beau paragraphe consacré à Empédocle, où se marque la rêverie simonienne de «conten[ir] en [soi] la présence de tout ce qui est vivant» (Tr., 127). Ce fantasme fait songer à ce que Bachelard, en 1949, dans La Psychanalyse du feu, nommera le " complexe d'Empédocle », désir de "brusquer le temps, de porter toute la vie à son terme, à son au-delà », manière de "tout perdre pour tout gagner», en une « mort cosmique »'. Un tel fantasme peut se comprendre comme le déploiement du sensualisme de Sartre dans La Nausée ${ }^{2}$, sensualisme dont Simon est profondément l'héritier, même si c'est plutôt du côté de la Phénoménologie de la perception que, toujours pour s'éloigner de Sartre, il cherchera explicitement appui. Mais de plus, ce fantasme n'est pas non plus sans analogie avec Le Sursis, où l'écrivain tente, usant d'un montage d'histoires fragmentées qui s'entrecroisent, d'écrire la totalité de l'Europe au moment de Munich. Sartre ne serait-il pas à cette époque en avance dans la réalisation du fantasme de Claude Simon ? Il lui « vole» et son thème privilégié (l'acte contre la loterie), et son fantasme préféré (écrire la totalité). C'est beaucoup, c'est assez pour se faire haïr...

Gallimard, rééd. coll. Folio/essais, 2000, p. 39, 41, 43.

On se souvient de l'éclatement du Cogito cartésien qui se produit en Roquentin. 
Que se passe-t-il dans La Corde raide, publié en 1947 ? Gide continue à être l'objet de vives railleries : il lui est reproché de " s'y connaî[tre] en fait de bons sentiments et de bonne littérature (il démontre par l'algèbre que l'ineffable pédérastie est la véritable gardienne de la sainte famille) » (CR, 127-128). Mais surtout, ce monologue rageur, qui voudrait évoquer Le Sous-sol, ou Le Bavard (publié en 1946), et ce même si la peinture y est donnée comme l'opposé absolu du bavardage, ce monologue donc me semble le lieu de la rupture la plus nette avec Sartre ${ }^{1}$. Petit inventaire en sept points.

1. Simon s'en prend à l'idée d'un choix qui engagerait dans la société : son narrateur regrette de vivre «[d]ans un univers où le social est à l'ordre du jour» $(C R, 29)$, et il considère que « [c]hoisir, c'est se différencier», donc préserver ou constituer sa solitude, et non pas se plonger dans le social. La notion d'engagement est parfois traitée par l'humour (pas très léger d'ailleurs) : le narrateur évoque « ces types qui s'inscrivent dans les partis politiques, qui s'engagent pour toute leur vie comme joueur de triangle dans les orphéons ou adhèrent à des ligues de morale " (175). Mais il arrive aussi que le romancier use d'un argument plus fin, qui consiste à dissocier radicalement engagement et liberté. Ainsi lorsqu'il médite sur « [1]a différence entre l'anarchiste de Barcelone et le soldat allemand» (56). Les Allemands par hypothèse ne concevraient pas la liberté ; or, « [s]i vous ne concevez pas la liberté, il vous est impossible de vous concevoir vous-même. Et ne pas se concevoir soi-même, douter de soi, c'est douter en même temps du reste du monde. À partir de ce moment, il n'y a plus qu'à s'engager, revêtir un uniforme et faire la guerre. » (57). On voit donc que l'engagement est la conséquence d'une inaptitude à saisir la liberté, d'une volonté de s'abriter contre le doute ; et non la forme concrète de la liberté, comme le voulait Sartre.

2. Simon critique l'idée même d'un choix : face au monde, il n'y a pas à choisir, pas à corriger, " seulement à prendre - tout à prendre " $(C R, 64)$, parce que «l'on n'arriverait jamais à se rassasier de cette

1 Même si sa forme peut évoquer les récits de vies publiés dans les premiers numéros des Temps modernes. 
somptueuse magnificence du monde ». Si la morale est l'envers du roman, c'est parce que la première est « une définition sélective et pratique du bien et du mal » (67), alors que le second entend ne rien se cacher de la vie. On voit de nouveau se dessiner cette vocation panique du roman selon Simon, cette pulsion de totalisation qui pousse l'écrivain à se laisser emplir et parcourir par le monde (« À la fin, je deviens cet univers apaisant, multiple et sans mesure où les fourmis sont aussi grandes que les maisons. » [63]), ou bien à devenir une foule («j'étais cette foule» [174]). C'est toujours le complexe d'Empédocle : se jeter dans le monde comme le philosophe dans l'Etna (il est fait allusion à cette légende p. 161). Dans La Corde raide, comme dans Le Tricheur, il me semble que Simon nourrit ce désir, sans avoir encore les moyens de le satisfaire sur le plan de l'écriture : il est, je le répète, techniquement en retard sur Le Sursis. Ce désir panique, il l'exprime à travers la vision du monde qu'il prête à Cézanne : celle d'un monde fait de résonances, où les choses vacillent et se pénètrent, s'enflant « de leur propre substance et de ce qu'elles absorbaient par une incessante osmose de leur immédiatt et lointain voisinage, une perpétuelle et obscure gestation, sourde, ardente» (122). Cette belle page qui décrit le surgissement d'une multiplicité labile et féconde, où les choses se donnent dans «leur présence en érection », on pourrait y voir la réponse à l'intuition de la contingence par Roquentin, où les arbres, moins glorieusement, sont des « verges lasses ». À la fin de La Corde raide, il est d'ailleurs question d'un arbre : le narrateur écoute « l'arbre palpiter et s'ouvrir, pousser ses ramures à travers [lui], [lui] emplissant les mains de ses feuilles» (137). Non plus donc un arbre (un marronnier) en face du sujet (Roquentin), et qui l'instruit de la contingence, mais un devenir-arbre du sujet (pour parler comme Deleuze).

3. Simon attaque la valeur de l'acte : le narrateur évoque "l'inanité dérisoire de tout acte » $(C R, 34)$; il n'y a pas lieu d'espérer qu'on puisse accélérer ou ralentir l'Histoire, personne "capricieuse et indépendante qui se désintéresse manifestement de ses soupirants » (125). Sartre est à l'évidence l'un de ces éconduits par l'Histoire.

4. Simon raille la théorie de la liberté : «Quelque chose de très abstrait probablement et d'une perfection dans le genre de la 
machine à vapeur à $100 \%$ de rendement» $(C R, 126)$. En vérité, pour lui, « la liberté d'un homme se dérobe et se passe superbement de commentaires et d'appréciations » (128).

5. Pas plus de bienveillance pour la théorisation de l'absurde: «[...] je pense que le non-sens est encore une invention des poètes et des philosophes. [...] Dire que ce monde est absurde équivaut à avouer que l'on persiste encore à croire en une raison. » $(C R, 64)$.

6. Simon ne croit pas à l'idée d'un chemin où l'on s'avancerait en bon équilibre. Le titre en ce sens, avec ses connotations nietzschéennes (Zarathoustra le danseur de corde), vaut comme une réponse à celui de Sartre, Les Chemins de la liberté: «Vous essayez tant bien que mal de continuer sur cette sacrée corde raide, manquant de vous casser la gueule à chaque pas [...]. On trouve dans toutes les bonnes librairies, à profusion, toutes les lois, recettes et dogmes infaillibles pour se bien conduire dans la vie et avancer en équilibre [...]. » $(C R, 61)$.

7. Enfin, Simon ne voue que mépris aux « abondants romans » $(C R, 28)$, ces "édifiantes œuvres morales » qui ne se distinguent guère de «cantiques révolutionnaires» (78), et dans lesquels, au terme d'un processus dont sont risibles « la continuité et la logique exemplaire» (174-175), les héros arrivent «au socialiste parfait et enthousiaste à la six cent vingt-troisième page» (175). Ces deux derniers griefs font trop peu de cas de l'inachèvement des Chemins de la liberté., lequel signifie entre autres que Sartre n'arrive pas à trancher entre l'intellectuel (Mathieu) et le militant (Brunei).

Malgré la constance de cette polémique, Simon devenu grand me semble un Sartre qui serait resté fidèle, du moins dans la métaphysique que suppose sa technique romanesque, à la contingence et à l'absurde. Par exemple, la guerre civile à Barcelone pendant l'été 36 apparaît au narrateur de La Corde raide, vue du côté des rouges, ou plutôt des noirs (anarchistes), comme le geste de « conduire ce hasardeux désordre qu'ils tentaient jusqu'à sa conclusion absurde» $(C R, 34)$. On découvre même une scène qui donne la vive impression que Simon réécrit encore La Nausée: scène de café, avec le narrateur en observateur solitaire et distancié, regardant les gens (dont certains «se demandent probablement aussi comme [lui] s'ils existent» 
[175-176]), écoutant la musique, et voyant la mort (176-177). La proclamation de solitude qui ouvre, ou peu s'en faut, La Corde raide, Roquentin aurait pu y souscrire: «Je pense que chaque homme est seul. [...] C'est tout seul, irrémédiablement, qu'il s'achemine, chargé de son passé qui n'appartient qu'à lui, inaliénable, vers sa mort qu'il devra affronter seul. »(20). Cependant, l'évolution de l'œuvre romanesque de Simon va dans le sens du chaos : "Partout la petite histoire et la grande Histoire, l'histoire individuelle et l'histoire collective s'imbriquent l'une dans l'autre sans parvenir à se libérer mutuellement de leur contingence respective. Les deux chaos s'interpénétrent et aucun n'est jamais en mesure de s'ordonner ni a fortiori d'ordonner l'autre en cosmos $»^{1}$. Il va donc découvrir la technique décomposée ou éclatée qui permet d'exprimer véritablement la contingence, le hasard, l'absurde. Simon peut apparaître comme un Sartre qui serait resté thématiquement jeune (fidèle à La Nausée), tout en mûrissant sur le plan formel (en dépassant Le Sursis dans l'art du désordre, ou plus exactement en situant ce désordre sur les plans de la mémoire et de la sensation). Mais à l'époque de La Corde raide, il reste encore à l'écrivain un long chemin à parcourir.

Gulliver (publié en 1952) représente une étape sur ce chemin. Sur le plan thématique, le romancier se dégage de l'influence sartrienne c'est un roman social, certes, comme l'a souligné Alastair Duncan, qui y voit « un hoquet» (a hiccup) dans la carrière de Simon, et le plus sartrien de ses romans ; pourtant, le thème même des jumeaux (l'un résista, l'autre fut de la Milice), implique une confusion des valeurs, donc un effort pour ne pas écrire de roman militant. Sur le plan du style, Simon commence à employer les alternatives (peutêtre. .. peut-être, soit que... soit que...), les longues parenthèses, et les participes présents caractéristiques de sa propre phrase.

Dans Le Sacre duprintemps (1954), on retrouve la même double référence, à Gide et à Sartre. À Gide : le jeune protagoniste se nomme Bernard, comme le héros des Faux-Monnayeurs, mais il estime que " l'Acte Gratuit » était à la mode du temps de son beaupère, lequel il a, comme de juste, rencontré au Luxembourg... 
À Sartre, puisque ledit beau-père se lance dans une tirade contre l'éthique existentialiste : "Ah oui la morale la vertu sans Dieu je suppose que tu as trouvé ça dans une de ces histoires finement transparentes de villes assiégées ou d'épidémies baptisées romans pour les commodités de la vente [...] » (SP, 57). Difficile de ne pas reconnaître ici Le Diable et le bon Dieu (1951) et La Peste (1947) ou D'État de siège (1948). L'épigraphe de la deuxième partie du roman, empruntée à Trotski, condamne le "point de vue de moraliste humanitaire sur le processus historique», qui est « tout ce qu'il y a de plus stérile ». Le narrateur de la première partie, quant à lui, se souvient avec dégoût de ses camarades de lycée ou de Fac qui sont « toujours à vous tanner pour vous amener à une réunion, vous faire signer une pétition, une protestation, un appel, ou quelque chose de ce genre » (77); et notamment d'un certain Abel, qui a appris à s'exprimer « sur ce ton réfléchi, un peu pédant, un peu pesant, usant de ce vocabulaire sélectionné qui permet de passer des envolées de meetings aux démonstrations philosophiques » (80). À la fin de la Deuxième partie, le beau-père de Bernard lui explique comment il fut, à Barcelone, en 1936, un «sacré boy-scout [...] en quête de la bonne action » (264), un "révolutionnaire de luxe» (265) : «Peutêtre avais-je trop lu Nietzsche, espéré, cru, rêvé corriger le sort, et ainsi, triché. Mettons si tu veux un peu de tout : la rédemption $\mathrm{du}$ hasard, la saoulerie de l'action, l'orgueil [...]». La critique de l'existentialisme sartrien est évidente : ce beau-père est un Mathieu Delarue, qui serait revenu de ses belles illusions sur la valeur de l'action ; quant à ce que Mathieu faisait effectivement dans L'Age de raison (se soucier d'un avortement, celui de sa compagne Marcelle), c'est exactement ce qui occupe le jeune Bernard, en une intrigue que Simon traite sur le mode dérisoire (le drame de Bernard est d'avoir perdu la bague qui lui a été confiée par la jeune femme enceinte... mais il la retrouve dans sa poche à la dernière page du roman).

Chose plus intéressante à noter, Claude Simon rejoint par le mot «triché » le titre de son premier roman, mais sur le mode de l'autocritique, puisqu'il y a inversion touchant la valeur de la tricherie elle était moyen de corriger le hasard par l'action, conformément à la formule de Nietzsche selon laquelle, même si c'est à tort, "nul 
vainqueur ne croit au hasard»(Le Gai Savoir, § 258); elle n'est plus que l'artifice et l'imposture qui s'attachent à l'acte : « [...] tout homme est obligé de tricher avec lui-même dès l'instant qu'il agit $[. .] ».(S P, 189)$. Pour finir, c'est à l'ontologie de Nietzsche que Simon semble s'en remettre, contre un Sartre devenu infidèle à $L a$ Nausée : ontologie pour laquelle «c'est l'incroyable hasard dont le jeu a jusqu'ici déterminé l'avenir de l'homme - un jeu où n'est intervenu ni la main ni même le " doigt de Dieu " " (Par-delà le Lien et le Mal, $\S 203)$. Si Claude Simon va devenir l'auteur non de romans militants mais de romans-poèmes, c'est parce qu'il en est venu à penser qu'un coup de dés toujours exhibera le hasard.

\section{Somme toute...}

Une fois les passions apaisées, et la place de chacun assurée dans l'histoire littéraire, que retenir de ce débat?

Je ne sais si Claude Simon, en choisissant le titre La Corde raide, se souvenait de ce passage des Voyages de Gulliver (roman qu'il aimait fort) qui raconte comment la danse de la corde raide était à Lilliput le moyen d'obtenir les plus grandes charges de l'État. Il semble plutôt vouloir suggérer que l'existence nous fait avancer sur une corde raide, sans aucune autre certitude que le vertige. Mais ce que je sais bien, c'est que la querelle entre Claude Simon et Sartre fait plus d'une fois penser à celle que décrit Swift entre les Gros-boutiens et les Petits-boutiens (rappelons que les Gros-boutiens, qui préfèrent, comme leur nom l'indique, ouvrir les œufs par le gros bout, ont été déclarés hérétiques à Lilliput). Car Sartre et Simon ont, au départ du moins, exactement les mêmes œufs romanesques dans les mains : le temps, le désordre du monde et de la mémoire, la contingence ou le hasard, le problème de l'acte...

Je me prends donc à rêver un peu. Sartre a donné une analyse virtuose des différents niveaux de la temporalisation, chez un individu et dans un roman, dans sa préface à L'Inachevé de Puig, en 1970, roman qui ne méritait pas tant d'honneur ${ }^{1}$. La relisant, je me disais : comme il eût été beau qu'il appliquât cette virtuosité

\footnotetext{
1 Reprise dans Situations, IX, ouvr. cité, p. 297-301.
} 
à la question du temps chez Claude Simon! En sens inverse, je me rappelle que Claude Simon avance une brève théorie des mots comme carrefours de sens (embrayeurs d'isotopies, dirions-nous) dans sa courte préface à Orion aveugle (1970), prenant l'exemple du mot tête, qui est commun à " $[\mathrm{u}]$ ne épingle, un cortège, une ligne d'autobus, un complot, un clown, un État, un chapitre» $(O A, 1182)$. Il aurait bien pu admettre que des mots-carrefours, ou des phrasesaiguillages, des " pivots structuraux », on en trouve en grand nombre dans Le Sursis, chef-d'œuvre méconnu, qui est lui aussi un roman du temps éclaté. Par exemple, lorsqu'on lit « la pendule sonna en face de lui, au-dessus de la glace », le pronom se réfère à la fois à Boris, dont il vient d'être question, et à Philippe, dont il va être question - tous deux se trouvant dans deux cafés différents, mais la phrase formant un facteur commun, et la transition ou l'aiguillage entre les deux séquences ${ }^{2}$.

Pour filer la métaphore ferroviaire, pourrait-on aller jusqu'à rapprocher Le Tramway de Claude Simon, et le tramway de $L a$ Nausée ? Le dernier texte « romanesque » de Simon rejoindrait ainsi, du moins par son titre, le premier roman de Sartre...

Mais coupons court. Trois remarques pour conclure. On peut d'abord regretter, dans ce débat, l'aigreur dont fait preuve Claude Simon. Je la décrirais volontiers en des termes empruntés à SainteBeuve, qui, en juin 1840, dans la Revue des deux mondes, blâmait les partisans de Victor Hugo d'intervenir en sa faveur tels " des gladiateurs en littérature» (c'est le titre de son article), et ajoutait que cela tenait au côté "cyclope» de Victor Hugo lui-même... Par transposition, on pourrait dire que Simon fait le gladiateur parce que Sartre fut plus d'une fois cyclope. Faut-il alors, toujours avec Sainte-Beuve, soutenir que la critique appartient, comme les mémoires et les souvenirs, aux "genres modérés », et plaider pour qu'y règnent la «bienveillance» et « une sorte de cordialité jusque

1 C'est le mot de Jean-Luc Seylaz, cité dans Sartre, Euvres romanesques, ouvr. cité, p. 1967.

2 bid., p. 977. 
dans les réserves $»^{1}$ ? Cet angélisme de l'humanitas s'accorde mal avec le caractère de nos deux héros, et avec leur époque. Je préfère, pour en finir avec Sainte-Beuve, rappeler son principe, si pertinent dans le cas présent, selon lequel « rien ne juge un esprit pour la portée et le degré d'élévation, comme de voir quel antagoniste et quel rival il s'est choisi de bonne heure. L'un est la mesure de l'autre $»^{2}$.

Cependant, la logique de l'affrontement, il se peut bien qu'elle appauvrisse la chose littéraire. C'était le sentiment de Paul Valéry, qui, dans ses Entretiens avec Frédéric Lefèvre (récemment réédités par les soins de Michel Jarrety, sous le titre Très au-dessus d'unepensée secrète), a abordé la question des disputes littéraires. Il trouve qu'elles manquent "de grâce et de résultat», et ce pour deux raisons. D'une part, si un romancier en attaque un autre, il semble «difficile d'échapper au soupçon de jalousie ou d'envie». D'autre part, et voici une belle idée, qui permet de dépasser cette « dialectique de la distinction ", entre nouveaux entrants et dominants, dont Bourdieu a voulu faire la théorie, " dans l'ordre de l'esprit, suggère Valéry, ne devraient paraître que des curiosités et des volontés si libres que chacun puisse concevoir l'œuvre d'autrui comme une expérience de soi-même. Il faudrait savoir se dire : ce mauvais ouvrage qui a paru, $c^{\prime} e s t$ moi qui l'ai fait, quoique dans un mauvais moment $»^{3}$. Voilà une leçon que Claude Simon aurait pu méditer: celle, le mot l'eût fait bondir, d'une espèce de communisme littéraire, propriété commune des chefs-d'œuvre et des ratages... Ou d'une égalité par l'œuvre virtuelle. Au fond, n'est-ce pas précisément parce que les romans de Sartre étaient une expérience de lui-même, d'un lui-même dont il voulait se déprendre, qu'il s'emporte contre eux ? Un double refoulé et vaincu, qu'il craint de voir revenir le hanter. Sartre comme le horla ou le dibbouk de Simon...

Dernière remarque, toute simple : pour moi, le plaisir demeure d'avoir, en lisant ces quatre premiers livres de Claude Simon, et malgré leurs incontestables faiblesses, vu naître un grand écrivain.

Pour la critique, Gallimard, coll. Folio/essais, 1992, p. 158, 139, 255.

2 Ibid., p. 157.

3 Très au-dessus d'une pensée secrète, Éditions de Fallois, 2006, p. 39. 
D'où il vient, et aussi d'où il revient. Comment il entre, lentement, durement, en possession de sa temporalité, et de ce qu'il a de plus précieux, et qui ne doit - songeons cependant à Proust... - presque rien à personne : sa phrase. 